\title{
DESCRIÇÃO DA CURVA DE CRESCIMENTO DE FRUTOS DO CAJUEIRO POR MODELOS NÃO LINEARES ${ }^{1}$
}

\author{
CARLOS ALBERTO MUIANGA², JOEL AUGUSTO MUNIZ³, \\ MICHERLANIA DA SILVA NASCIMENTO ${ }^{4}$, TALES JESUS FERNANDES 5 , \\ TACIANA VILELLA SAVIAN ${ }^{6}$
}

RESUMO - O objetivo do trabalho foi avaliar o ajuste dos modelos Gompertz e Logístico, com estrutura de erros independentes e autorregressivos, no desenvolvimento de frutos de caju, com base em medidas de comprimento e largura do fruto, tomados ao longo do tempo. A estimação dos parâmetros foi feita por meio de rotinas no software R, utilizando-se do método dos mínimos quadrados e o processo iterativo de GaussNewton. Os modelos foram comparados pelos critérios: coeficiente de determinação ajustado $\left(\mathrm{R}_{\mathrm{aj}}^{2}\right)$, desvio padrão residual (DPR), critério de informação Akaike (AIC) e critério bayesiano de Schwarz (BIC). Para os dois modelos, os dados apresentaram autocorrelação residual positiva, tanto para a variável comprimento como para a largura do fruto, descrita por processo autorregressivo de primeira ordem. Em geral, por todos critérios de avaliação da qualidade de ajuste, os dados se ajustaram ao modelo Logístico com uma estrutura autorregressiva da primeira ordem, havendo no entanto superestimação do tamanho do fruto nas últimas idades, tanto no crescimento em comprimento $(\mathrm{cm})$ como em largura $(\mathrm{cm})$.

Termos para indexação: Modelo de crescimento. Medidas biométricas. Regressão não linear. Cajú.

\section{DESCRIPTION OF THE GROWTH CURVE OF CASHEW FRUITS IN NONLINEAR MODELS}

\begin{abstract}
The objective of this study was to evaluate the adjustment of gompertz and logistic models, with independent and autoregressive error structure in cashew fruits growth, based on the length and width measurements of the fruit, taken through the time. The estimation of the parameters was done in $\mathrm{R}$ software routines using the method of minimal squares and the interactive process of Gauss-Newton. The models were compared by the following criteria: the adjusted coefficient of determination, residual standard deviation, Akaike information criterion and Schwarz Bayesian criterion. For both models, the data showed positive residual autocorrelation for both variables: length and width of the fruit, described in autoregressive process of order one. In general, both models adjusted to the data, however there was an overestimation of the fruit size at the late stages. The logistic model was the most adequate to describe the cashew fruit growth in length and width.
\end{abstract}

Index terms: Growth model, Biometric measurements, Nonlinear regression, cashew.

\footnotetext{
'(Trabalho 295-14). Recebido em: 17-11-2015. Aceito para publicação em: 23-04-2015.Programa de Pós-Graduação em Estatística e Experimentação Agropecuária, Departamento de ciências exactas-UFLA/Lavras-MG. E-mail: muiangacarlo@gmail.com 2DEX/UFLA, Cx. P. 3037, 37-200-000, Lavras-MG. E-mail: joamuniz@dex.ufla.br 32DEX/UFLA, Cx. P. 3037, 37-200-000, Lavras-MG. E-mail: micherlania@bol.com.br ${ }^{4}$ DEX/UFLA, Cx. P. 3037, 37-200-000, Lavras-MG. E-mail: talesest@yahoo.com.br ${ }^{5}$ Departamento de Ciências Exatas, ESALQ/USP, Piracicaba-SP. . E-mail: tvsavian@usp.br
} 


\section{INTRODUÇÃO}

O cajueiro (Anacardium occidentalle L.) é uma planta da família Anacardiaceae, originária das regiões Norte e Nordeste do Brasil. Trata-se de uma frutífera tropical na qual a comercialização dos produtos envolve intensa atividade econômica com expressiva movimentação de recursos. Além da amêndoa, o cajueiro fornece outros produtos aceitos pelo mercado, destacando-se o líquido da casca de castanha, o suco, a polpa, os doces, dentre outros derivados (MENDONÇA; MEDEIROS, 2011).

A planta de caju está difundida no mundo e tem a castanha, como principal produto. Entre os países com maior produção de castanha destacamse İndia, Brasil, Moçambique, Tanzânia, Quênia e Vietnã. No Brasil, a produção de castanha de caju concentra-se, principalmente, no Ceará, Piauí e Rio Grande do Norte (SEBRAE, 2005).

A cultura do caju tem grande importância econômica em função da elevada demanda do mercado por seus produtos; castanha e pedúnculo (PARENTO; SANTOS, 1970). O fruto é composto do pedúnculo e da castanha. Em termos botânicos, o verdadeiro fruto é a castanha, enquanto que o pedúnculo é o pseudofruto, que é comestível na forma "in natura" ou industrializada (FILHO SOUZA et al., 1989). O pedúnculo representa aproximadamente 90 $\%$ do peso do fruto de caju, enquanto a castanha e a amêndoa constituem os $10 \%$ restantes (PAIVA et al., 2000). A amêndoa da castanha é o principal produto do cajueiro sendo rica em proteínas, lipídios, fósforo, ferro, fibras, gordura insaturada e outros minerais (GAZZOLA et al., 2006).

Durante o desenvolvimento, a castanha e o pedúnculo crescem de forma independente. Após a antese o processo de crescimento da castanha é rápido, atingindo o tamanho máximo em torno de 30 dias. O pedúnculo inicialmente cresce de forma lenta, acelerando o crescimento após 30 dias, atingindo o tamanho máximo próximo dos 50 dias, quando em geral está maduro com coloração entre amarelo e vermelho (PRATT e MENDONZA, 1986; YAHIA, 2001).

A avaliação do processo de crescimento e desenvolvimento das plantas e dos frutos, medido pelo acúmulo de biomassa ao longo do tempo é fundamental para o manejo adequado da cultura, possibilitando estabelecer estratégias de tratos culturais ou mesmo de práticas úteis pós-colheita e armazenamento (SANTOS et al., 2009; TERRA et al., 2010). Além disto, o estudo do padrão de crescimento do fruto auxilia na definição de procedimentos na colheita, de técnicas de manuseio e conservação no pós-colheita e de índices de maturação e de qualidade do fruto (ARAÚJO NETO et al., 2001).

Diversos modelos estatísticos podem ser utilizados no estudo envolvendo o processo de crescimento no sistema de produção vegetal. Os modelos não lineares geralmente fornecem melhor ajuste que os modelos lineares em estudos de crescimento, tendo ainda a vantagem de fornecer estimativas de parâmetros com interpretação biológica (MAZZINI et al., 2003; MENDES et al., 2008; FERNANDES et al., 2014). Os modelos não lineares Gompertz e Logístico foram ajustados com resultados satisfatórios para descrever o crescimento de frutos de coqueiro anão verde (PRADO et al., 2013) e de frutos de tamareira anã (TERRA et al., 2010).

A estimação dos parâmetros em trabalhos com modelos não lineares, em geral é feita por meio da minimização da soma de quadrados dos erros, o que leva a um sistema de equações normais não lineares, o qual não tem solução explícita, sendo necessária a utilização de processos iterativos para obtenção das estimativas (SOUZA, 2007; DRAPER E SMITH, 1998). Diversos métodos iterativos são utilizados, destacando-se o de Gauss Newton (SAVIAN e MUNIZ, 2007; PEREIRA et al.; 2005; SOUZA et al., 2010).

Em estudos de regressão é comum admitir que os erros envolvidos no processo de estimação são independentes, o que não ocorre quando se trabalha com séries cronológicas (MORETIN e TOLOI, 2004). No caso de haver dependência entre os erros, as estimativas obtidas podem ser viesadas, com valores abaixo ou acima do verdadeiro valor do parâmetro (GUEDES et al., 2004; MAZZINI et al., 2005; PEREIRA et al., 2005). Em estudo do crescimento dos diâmetros longitudinal e transversal do fruto de coqueiro anão verde, Prado et al., (2013) obtiveram melhor ajuste dos modelos Gompertz e Logístico quando consideraram estrutura autocorrrelacionada de ordem 1 (AR1).

Este trabalho teve por objetivo avaliar o ajuste dos modelos Gompertz e Logístico, aos dados do crescimento em comprimento e largura do fruto do cajueiro, considerando erros independentes e com estrutura autoregressiva.

\section{MATÉRIAL E MÉTODOS}

Os dados utilizados para o ajuste dos modelos foram obtidos em um experimento realizado na Unidade de Pesquisa da Pacajus da Empresa de Pesquisa Agropecuária do Ceará, envolvendo 
o cajueiro do tipo anão precoce, com 9 anos de idade (ALMEIDA et al., 1987). Os pesquisadores acompanharam o desenvolvimento dos frutos medindo-se o comprimento e a largura. Foram inspecionadas diversas inflorescências, marcandose inicialmente 100 flores fecundadas. As medidas foram realizadas com o auxílio de paquímetro, sendo a primeira medição efetuada seis dias após a fecundação das flores. As medições seguintes foram realizadas a cada seis dias até a idade de 36 dias, a partir da qual passou-se a medir a cada quatro dias até a idade de 52 dias, quando ocorreu a queda natural dos frutos, totalizando-se dez idades.

Os modelos não lineares, Logístico (1) e Gompertz (2) foram utilizados para estimar os parâmetros da curva de crescimento dos frutos de caju, com base nas seguintes equações: $\mathrm{Y}_{\mathrm{i}}=\mathrm{A} /\{1+$ $\left.\exp \left(\mathrm{B}-\mathrm{Kt}_{\mathrm{i}}\right)\right\}+\mathrm{u}_{\mathrm{i}}(1)$ e $\mathrm{Y}_{\mathrm{i}}=\mathrm{A} \exp /\left\{-\exp \left(\mathrm{B}-\mathrm{Kt}_{\mathrm{i}}\right)\right\}$ $+u_{i}(2)$, em que: $u_{i}={ }_{1} u_{i-1}+\ldots \rho_{p} u_{i-p+} \varepsilon_{i}, \operatorname{com~} i=1,2, \ldots, n$ e $\mathrm{n}$ o número de tempos em que foram realizadas as medidas de crescimento; $u_{i}$ é o resíduo do ajuste do i-ésimo tempo; $\rho_{1}$ é o parâmetro autoregressivo de ordem $1 ; \mathrm{u}_{\mathrm{i}-1}$ é o resíduo do ajuste do tempo imediatamente anterior a i-ésima medida; $\rho_{p}$ é o parâmetro autoregressivo de ordem $\mathrm{p} ; \rho_{\mathrm{i}-\mathrm{p}}$ é o resíduo do ajuste em $\mathrm{p}$ tempos anterior a i-ésima medida, $\varepsilon_{\mathrm{i}}$ é o resíduo branco, com distribuição normal $\mathrm{N}(0$, $\left.\sigma^{2}\right)$. No caso de erros independentes, os parâmetros $\rho_{1}$ são nulos, então $u_{i}=\varepsilon_{i}$ (MAZZINI et al., 2005; PRADO et al., 2013).

Para as equações (1) e (2), Yi descreve o valor médio do comprimento e da largura do fruto de caju no tempo $\mathrm{t}$ em dias; A expressa o valor assíntótico das medidas, ou seja, o valor das variáveis quando o fruto está totalmente desenvolvido; B representa o parâmetro de locação, sem interpretação biológica, $\mathrm{k}$ indica a taxa de crescimento do fruto; t. refere-se ao tempo na i-ésima medição, dado em dias após a fecundação, com $\mathrm{i}=1,2, \ldots, 10$. Para 0 modelo Gompertz, o ponto de inflexão é $\mathrm{Y}=\mathrm{A} /$ $\mathrm{e}=\mathrm{A} / 2,7182$, indicando que o fruto tem taxa de crescimento máxima antes de atingir a metade do desenvolvimento máximo. Para o modelo Logístico, o ponto de inflexão é $\mathrm{Y}=\mathrm{A} / 2$, indicando tratar-se de um modelo simétrico, ou seja, a taxa de crescimento é máxima quando o fruto atinge metade do seu desenvolvimento (TSOULARIS e WALACE, 2002).

Para o ajuste dos modelos aos dados foi utilizado o software R (R DEVELOPMENT CORE TEAM, 2013) estimando-se os parâmetros por meio dos pacotes lmtest (Testing linear Regression Models) descrito por Hothorn et al., (2014), nlstools (Tools for Nonlinear Regression Analysis) descrito por Baty (2014) e qper (Modelling and Analysis of real-time PCR data) descrito por Spiess (2014) disponíveis na versão 3.12. O método de estimação utilizado o de mínimos quadrados generalizados.

Para testar a homogeneidade de variância dos resíduos foi utilizado o teste de Breusch e Pagan, conforme descrito em Breusch e Pagan (1979) Para verificar a normalidade dos erros foi aplicado o teste de Shapiro-Wilk, de acordo com procedimento apresentado em Shapiro e Wilk (1965). A verificação da presença de autocorrelação residual foi feita da seguinte forma: inicialmente, fez-se o ajuste dos modelos considerando erros independentes. Após o ajuste foi estimado o vetor dos resíduos, aplicandose posteriormente o teste de Durbin-Watson (1950), verificando-se a hipótese de que os resíduos são não correlacionados, contra a alternativa de que existe um processo autoregressivo de primeira ordem. Quando o teste foi significativo, o parâmetro de autocorrelação foi estimado com base no vetor de resíduos (CARNEIRO et al.,2014)

A comparação dos modelos levou em consideração a interpretação agronômica das estimativas dos parâmetros e ainda a qualidade do ajuste com base nos seguintes critérios:

1. Desvio Padrão residual (DPR), calculado pela expressão $\mathrm{DPR}=\sqrt{\mathrm{QME}}$, sendo $\mathrm{QME}$ o quadrado médio residual; 2 . Coeficiente de determinação ajustado $\left(\mathrm{R}^{2}{ }_{\mathrm{aj}}\right)$, obtido por:

$R^{2} a=1-\left[\frac{\left(1-R^{2}\right)(n-i)}{n-p}\right]$, sendo $R^{2}$ o coeficiente

de determinação não ajustado, n o número de tempos em que foram feitas medições e p o número de parâmetros do modelo. 3. Critério de informação de Akaike (AKAIKE, 1974), dado por: AIC = $-2 \log \mathrm{L}(\widetilde{\theta})+2 p$, em que: L $(\widetilde{\theta})$ é o máximo da função de verossimilhança, $\mathrm{p}$ é o número de parâmetros no modelo e log é o operador logaritimico natural. O primeiro termo é uma bonificação por uma melhor adequação aos dados, e o segundo termo é uma penalização. Sendo que o melhor modelo escolhido será aquele que apresenta menor valor de AIC, 4. Critério Bayesiano de Schwarz (BIC) obtida pela expressão: $\mathrm{BIC}=-2 \log \mathrm{L}(\widetilde{\theta})+\operatorname{plog}(\mathrm{n})$, em que $\mathrm{L}$ $(\widetilde{\theta})$ é o máximo da função de verossimilhança, $p$ é o número de parâmetros no modelo e n é o número de observações utilizadas na estimação do modelo em estudo e log é o operador logaritimico natural. O critério de informação bayesiano (BIC) é definido como a estatística que maximiza a probabilidade de se identificar o modelo mais adequado dentre os avaliados. Quanto menor o valor do critério de informação, melhor será o modelo.

Após o ajuste dos modelos, foram calculados 
os intervalos de confiança (IC) de $95 \%$ para as estimativas dos parâmetros com base nas estimativas de erro padrão obtida a partir das estimativas da matriz assintótica de variâncias e covariâncias conforme a seguinte expressão:

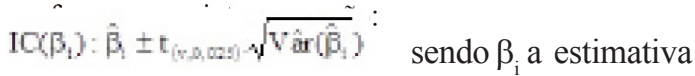
do parâmetro $\beta_{\mathrm{i}}, \mathrm{t}_{(\mathrm{v}, 0.025)} \mathrm{o}$ valor na distribuição de $\mathrm{t}$ com $\mathrm{v}$ graus de liberdade e área 0,025 acima e $\sqrt{\operatorname{ar}\left(\beta_{\mathrm{i}}\right)}$ a estimativa do erro-padrão da estimativa do parâmetro $\beta_{\mathrm{i}}$ obtido da diagonal da matriz assintótica de variâncias e covariâncias.

\section{RESULTADOS E DISCUSSÃO}

Na tabela 1 estão apresentados os resultados da análise de resíduos, com base nas significâncias para os testes Shapiro-Wilk (SW), Breusch-Pagan (BP) e Durbin-Watson (DW). De acordo com os resultados dos testes verificou-se que os pressupostos de homogeneidade de variâncias e de normalidade dos erros ocorreram para as variáveis comprimento $(\mathrm{cm})$ e largura $(\mathrm{cm})$ dos frutos para os dois modelos, $(\mathrm{p}>0,05)$. Como os resultados do teste DurbinWatson indicaram, as medidas de comprimento $(\mathrm{cm})$ e largura $(\mathrm{cm})$ apresentaram presença de autocorrelação residual $(\mathrm{p}<0,05)$ que foi modelada por um processo autoregressivo de primeira ordem (AR1) tanto no modelo Gompertz, quanto no Logístico. Estes resultados concordam com os estudos de Fernandes et al., (2014), e Prado et al., (2014) que encontraram erros autocorrelacionados quando avaliaram o ajuste dos modelos Gompertz e logístico a dados de crescimento de frutos de café e de coco anão, respectivamente.

$\mathrm{Na}$ tabela 2 e 3 estão apresentadas as estimativas dos parâmetros de ajuste dos modelos Gompertz e logístico, para os dados das variáveis comprimento e largura dos frutos de caju tomados em 10 tempos, considerando a estrutura de erros independentes e autoregressivos.

A incorporação do processo AR1 no ajuste dos modelos da variável resposta comprimento $(\mathrm{cm})$ (tabela 2) resultou em alterações muito pequenas nas estimativas dos parâmetros do modelo, quando comparado ao modelo sem AR. No modelo Gompertz as estimativas do parâmetro B e K sofreram uma redução de $0,08 \%$ e $0,06 \%$ respectivamente, enquanto que a estimativa do parâmetro $\mathrm{A}$ não se alterou. No modelo Logístico, a incorporação do AR1 resultou em uma redução nas estimativas dos parâmetros B e K de 0,02 e 0,04 \%. A incorporação do processo AR1 no ajuste dos modelos da variável resposta largura $(\mathrm{cm})$ resultou em alterações muito pequenas nas estimativas do modelo Gompertz, sendo que as estimativas dos parâmetros $\mathrm{A}, \mathrm{B}$ e K sofreram uma redução de $0,069 \%, 0,07 \%$ e 0,083 $\%$ respectivamente. No modelo Logístico não se verificou alteração das estimativas nos parâmetros.

Para a amplitude dos intervalos de confiança, no crescimento em comprimento, a incorporação do processo AR1 no ajuste dos modelos resultou numa expressiva redução quando comparado ao modelo sem AR. Sendo que a maior alteração foi observada no parâmetro B do modelo Gompertz que sofreu uma redução de $22 \%$ na amplitude. Os parâmetros A e K sofreram uma redução de $15 \%$ e $19 \%$ respectivamente. No modelo logístico a maior alteração foi observada na amplitude do parâmetro B que sofreu uma redução de 19 \%. Nos parâmetros A e K a amplitude de redução do intervalo de confiança foi de $15 \%$.

No crescimento em largura $(\mathrm{cm})$, a maior redução da amplitude do intervalo de confiança foi verificada no parâmetro B (tabela 3 ), tanto no modelo logístico como no Gompertz. A redução foi de $23 \%$ e $19 \%$ para modelo Logístico e gompertz respectivamente. No modelo Gompertz, a redução na amplitude dos parâmetros A e C foram de $15 \%$ e $21 \%$ respectivamente, enquanto que no modelo logístico a redução foi de $15 \%$ e 18 \% nos parâmetros A e C, respectivamente. É nítido que o intervalo de confiança com AR1 tem amplitude menor e portanto, a estimativa é mais precisa. Resultados similares foram encontrados por Mazzini et al., (2005), Pereira et al., (2005), Mendes et al., (2008), Terra et al., (2010) e Fernandes et al., (2014), no ajuste de modelos não lineares, considerando estrutura autoregressiva de primeira ordem para o vetor residual que também obtiveram estimativas mais precisas dos parâmetros.

$\mathrm{Na}$ tabela 4 estão apresentados os valores das medidas de qualidade do ajuste dos modelos Gompertz e logístico, para os dados das variáveis comprimento e largura dos frutos de caju considerando a estrutura de erros independentes e autoregressivos. Em relação a comparação dos modelos considerando a estrutura AR1, com base nos valores das medidas de qualidade de ajuste apresentadas na tabela 2 , podese verificar que os dados apresentaram melhores ajustes ao modelo Logístico com uma estrutura autoregressiva da primeira ordem no crescimento tanto em comprimento $(\mathrm{cm})$ e largura $(\mathrm{cm})$. O modelo Logístico AR1 evidenciou superioridade em relação ao modelo Gompertz, pois, apresentou menores valores do $\mathrm{R}^{2}$,j , DPR, AIC e BIC. para as duas variáveis. 
Os ajustes dos dados, de comprimento $(\mathrm{cm})$ e largura $(\mathrm{cm})$, aos modelos Logístico e gompertz podem ser visualizados nas figuras 1 e 2 . Observa-se que o modelo Logístico AR1 adequouse perfeitamente nas primeiras observações (até vigésimo quarto dia), sendo que o Logístico adequouse melhor devido a simetria em relação ao ponto de inflexão. Observando as figuras 1 e 2 é possível verificar um padrão de crescimento sigmoidal do comprimento e largura do fruto. $\mathrm{Na}$ descrição do crescimento observa-se uma redução do crescimento a partir do quadragésimo segundo dia após observada a fecundação (últimas três observações), verificandose uma redução de crescimento em largura e comprimento. Isto se deve, provavelmente, devido ao fato dos frutos já terem atingido a maturação fisiológica. Estes resultados concordam com Carvalho e Nakagawa (2000), que afirmam que a partir da maturação fisiológica ocorre a diminuição dos teores de água e das dimensões do fruto. Este processo inicia a partir do período em que a castanha está madura e seca com o pedúnculo vermelho de acordo com a escala de maturação sugerida por Alves et al., (1999) para clones avermelhados. Resultados semelhantes foram encontrados por Pratt e Mendonza (1980), que relatam que após o crescimento máximo do fruto ocorre redução da noz em até $40 \%$ bem como endurecimento da casca.

Com respeito à estabilização do crescimento os resultados indicaram que o comprimento começa a se estabilizar em torno de 35 dias após observada a fecundação, o que ocorreu aproximadamente no dia 27 de Outubro de acordo com a data em que foi observada a fecundação, com o fruto apresentando um valor estimado de $3,45 \mathrm{~cm}$. Já a largura começa a se estabilizar em torno de 33 dias após observada a fecundação, aproximadamente no dia 23 de Outubro com o fruto apresentando um valor estimado de 2,70 $\mathrm{cm}$. Resultados semelhantes foram encontrados por Pratt e Mendonza (1980), em estudos de crescimento do fruto do cajueiro, onde o crescimento máximo foi alcançado em torno dos 30 dias, após a antese. Os resultados concordam também com Almeida et al., (1987) e Filgueiras et al., (1999), que em estudo de crescimento do fruto de cajueiro, observaram crescimento máximo entre o trigésimo e o trigésimo sexto dia após a fecundação.

Considerando a estrutura residual adequada (modelo logístico autoregressivo da primeira ordem), no ajuste do comprimento, o ponto de inflexão ocorreu quando o fruto alcançou metade do seu crescimento, ou seja: $\frac{\mathrm{A}}{2}=\frac{3,4461}{2,000} \approx 1,72 \mathrm{~cm}$, no tempo $\frac{\mathrm{B}}{\mathrm{k}}=\frac{3,9767}{0,2478} \approx 16$ dias após observada a fecundação.
No crescimento em largura, o ponto de inflexão ocorreu quando o fruto alcançou $1,36 \mathrm{~cm}$, no décimo sexto dias após observada a fecundação, indicando que o crescimento do fruto do cajueiro é simétrico, isto é, o período em que a taxa de crescimento do fruto é decrescente e crescente durante o desenvolvimento do fruto é equivalente. 
TABELA 1- Estatísticas de testes e nível de significância entre parênteses para o teste Shapiro-Wilk (SW), Breusch-Pagan (BP) e Durbin-Watson (DW) na análise dos resíduos estimados após o ajuste dos modelos Gompertz e Logístico aos dados das medidas de comprimento e largura dos frutos de cajú, tomados em 10 tempos.

\begin{tabular}{llll}
\hline \multirow{2}{*}{ Varíavel } & \multirow{2}{*}{ Teste } & \multicolumn{2}{c}{ Modelo } \\
\cline { 3 - 4 } Comprimento & SW & $0,9006(0,2226)$ & $0,9447(0,6066)$ \\
& BP & $2,2295(0,3280)$ & $3,7729(0,1516)$ \\
& DW & $0,7153(0,0020)$ & $0,5660(0,0001)$ \\
\hline \multirow{3}{*}{ Largura } & SW & $0,9053(0,2500)$ & $0,9745(0,4938)$ \\
& BP & $2,0546(0,3580)$ & $3,1596(0,2010)$ \\
& DW & $0,7030(0,0020)$ & $0,5510(0,0020)$ \\
\hline
\end{tabular}

SW - Shapiro-Wilk, BP - Breusch-Pagan e DB - Durbin-Watson.

TABELA 2- Estimativas do ajuste dos dados aos modelos não-lineares (Gompertz e logístico) no crescimento em comprimento $(\mathrm{cm})$ de frutos de caju e na amplitude dos intervalos de confiança, considerando estruturas de erros independentes e autoregressivos da primeira ordem (AR1)

\begin{tabular}{|c|c|c|c|c|c|c|c|c|}
\hline \multirow{3}{*}{$\begin{array}{l}\text { Variáveis } \\
\text { Parametros } \\
\text { Comprimento }\end{array}$} & \multicolumn{7}{|c|}{ Modelo } & \multirow[b]{3}{*}{ Amplitude } \\
\hline & \multicolumn{3}{|c|}{ GOMPERTZ } & & \multicolumn{3}{|c|}{ GOMPERTZ AR1 } & \\
\hline & LI & Estimativa & LS & Amplitude & LI & Estimativa & LS & \\
\hline A & 3,2023 & 3,4674 & 3,7641 & 0,5618 & 3,2284 & 3,4674 & 3,7065 & 0,4781 \\
\hline B & 1,3335 & 2,3644 & 4,2461 & 2,9126 & 1,2276 & 2,3624 & 3,4973 & 2,2697 \\
\hline K & 0,1098 & 0,1763 & 0,3001 & 0,1903 & 0,0986 & 0,1762 & 0,2538 & 0,1552 \\
\hline \multirow[t]{3}{*}{$\rho_{1}$} & - & - & - & & & 0,4519 & & \\
\hline & \multicolumn{3}{|c|}{ LOGÍSTICO } & & \multicolumn{3}{|c|}{ LOGÍSTICO AR1 } & \\
\hline & LI & Estimativa & $\mathrm{LS}$ & Amplitude & LI & Estimativa & LS & Amplitude \\
\hline A & 3,2383 & 3,4460 & 3,6618 & 0,4235 & 3,2667 & 3,4461 & 3,6255 & 0,3588 \\
\hline B & 2,7124 & 3,9776 & 6,1066 & 3,3942 & 2,6025 & 3,9767 & 5,3509 & 2,7484 \\
\hline K & 0,1729 & 0,2479 & 0,3737 & 0,2008 & 0,1623 & 0,2478 & 0,3333 & 0,1710 \\
\hline$\rho_{1}$ & - & - & - & & & 0,51 & & \\
\hline
\end{tabular}

LI-limite inferior, LS-limite superior 
TABELA 3 - Estimativas do ajuste dos dados aos modelos não-lineares (Gompertz e logístico) no crescimento em largura $(\mathrm{cm})$ de frutos de caju e na amplitude dos intervalos de confiança, considerando estruturas de erros independentes e autoregressivos da primeira ordem (AR1).

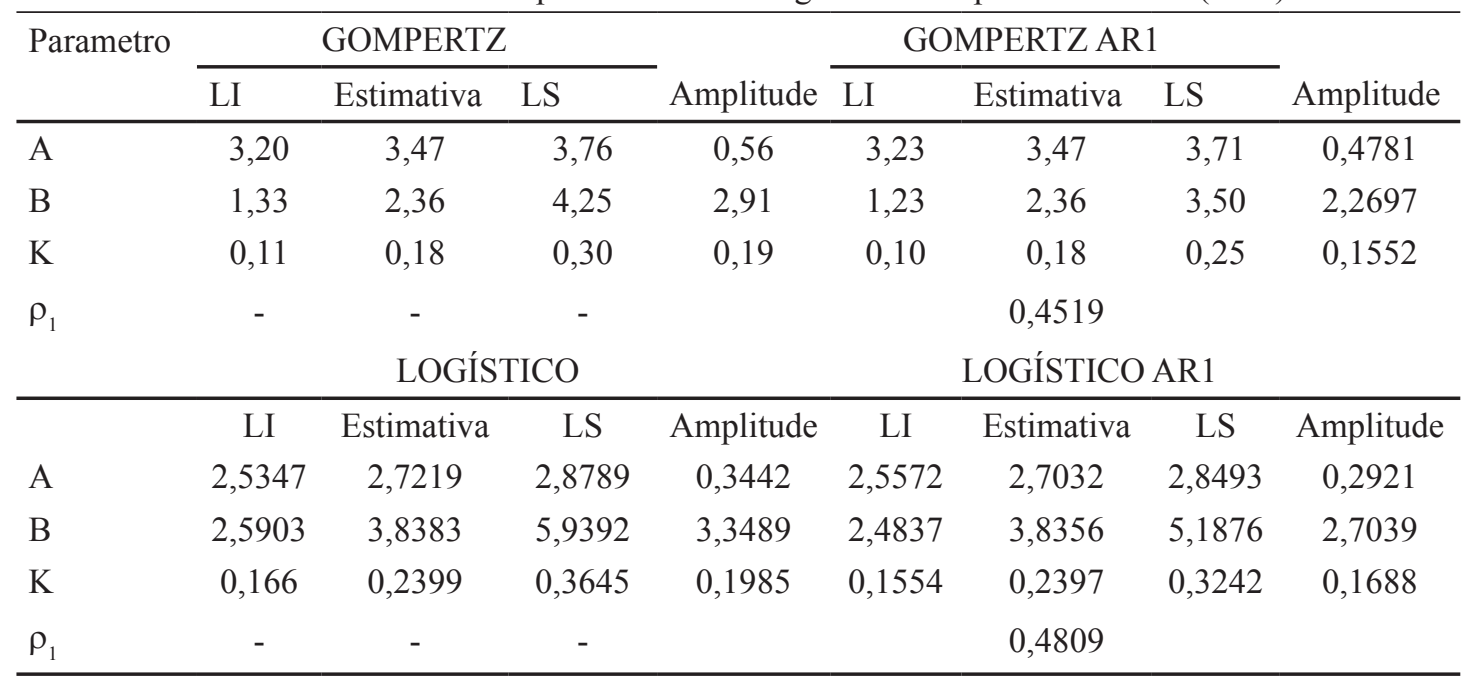

LI-limite inferior, LS-limite superior.

TABELA 4 - Avaliadores de qualidade de ajuste dos modelos gompertz e logístico, para os dados das variáveis comprimento $(\mathrm{cm})$ e largura $(\mathrm{cm})$

\begin{tabular}{llcccc}
\hline Variável & Modelo & $\mathrm{R}_{\mathrm{aj}}^{2}$ & $\mathrm{DPR}$ & $\mathrm{AIC}$ & $\mathrm{BIC}$ \\
\hline \multirow{4}{*}{ Comprimento } & Gompertz & 0,9605 & 0,2556 & 5,5317 & 6,7420 \\
& Logístico & 0,9703 & 0,2102 & 1,6198 & 2,8302 \\
& Gompertz AR1 & 0,9605 & 0,2556 & 7,5317 & 9,0447 \\
& Logístico AR1 & 0,9703 & 0,2102 & 3,6198 & 5,1328 \\
\hline \multirow{3}{*}{ Largura } & Gompertz & 0,9575 & 0,2057 & 1,1843 & 2,3946 \\
& Logístico & 0,9681 & 0,1696 & $-2,6786$ & $-1,4682$ \\
& Gompertz AR1 & 0,9575 & 0,2057 & 3,1843 & 4,6972 \\
& Logístico AR1 & 0,9780 & 0,1696 & $-0,6785$ & 0,8344 \\
\hline
\end{tabular}

$\overline{\mathrm{R}^{2} \text { aj }}$-coeficiente de determinação ajustado, DPR-desvio padrão residual, AIC-critério de informação Akaike e BIC-critério bayesiano de Schwarz. 


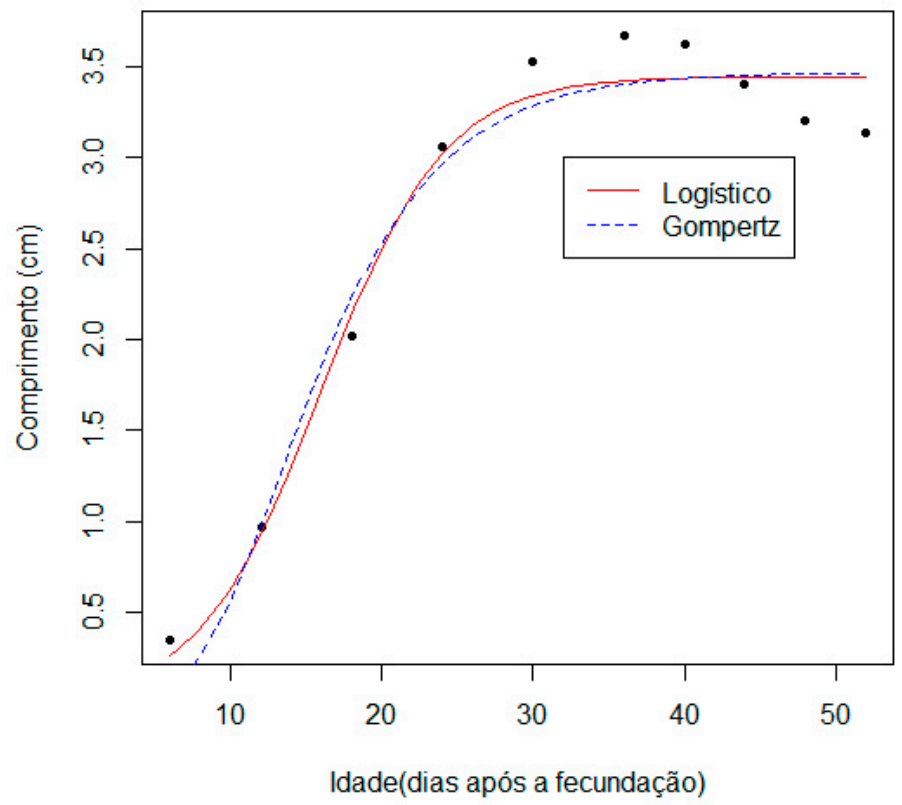

FIGURA 1-Modelos Logístico e gompertz na descrição do crescimento em comprimento do cajueiro em função do tempo com estrutura de erros autorregressivos de primeira ordem.

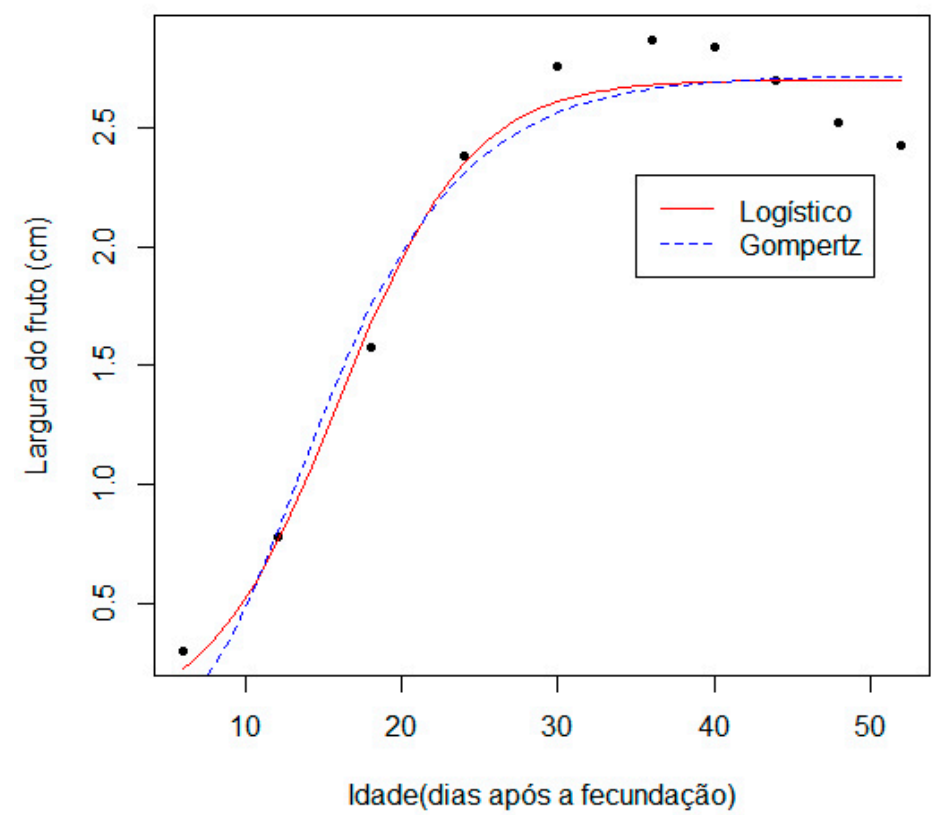

FIGURA 2- Modelos Logístico e gompertz na descrição do crescimento em largura do fruto do cajueiro em função do tempo com estrutura de erros autorregressivos da primeira ordem. 


\section{CONCLUSÕES}

Os modelos Logístico e gompertz apresentaram erros dependentes para os dados de comprimento $(\mathrm{cm})$ e largura $(\mathrm{cm})$, que foram ajustados por um processo autoregressivo de primeira ordem (AR1). O ajuste dos dados aos modelos, considerando a estrutura de autocorrelação, apresentou estimativas de parâmetros com maior precisão.

O modelo Logístico com uma estrutura autoregressiva da primeira ordem descreveu o crescimento dos frutos de cajueiro com bons indicadores de qualidade de ajuste, mas superestimou o tamanho do fruto nas últimas idades.

$\mathrm{O}$ crescimento em comprimento $(\mathrm{cm})$ e largura $(\mathrm{cm})$ estabilizou-se em torno de 33 e 35 dias após a fecundação, respectivamente, com o fruto apresentando um valor estimado de $2,70 \mathrm{~cm}$ e $3,45 \mathrm{~cm}$ para as duas variáveis.

\section{AGRADECIMENTOS}

Ao Conselho Nacional de Desenvolvimento Científico e Tecnológico (CNPq), pela concessão da bolsa e ao Programa de Pós-Graduação em Estatística e Experimentação Agropecuária, da Universidade Federal de Lavras pelo apoio prestado durante a pesquisa.

\section{REFERÊNCIAS}

AKAIKE, H.A. New Look at the Statistical Model Identification. IEEE Transactions on Automatic Control, Minato-ku, v.19, n.6, p.716-723, 1974.

ALMEIDA, I.L.D. et al. Estudo sobre o crescimento do fruto e pseudo-Fruto do cajueeiro (Anarcadium occidentalle L.) do tipo anão precoce. Revista Brasileira de Fruticultura, Jaboticabal, v.9, n.3, p.21-30, 1987.

ALVES, R.E.; BEZERRA, F.C.; ABREU, F.A.P. Development and maturation of the apple of early dwarf cashew tree CCP 76. Acta Horticulturae, The Hague, n.485, p.230-255, 1999.

ARAUjO NETO, S.E. et al. Desenvolvimento de frutos de sapotizeiro. Revista Brasileira de Fruticultura, Jaboticabal, v.23, n.1, p.25-29, 2001.
BATY, F. Package 'nlstools' .Tools for Nonlinear Regression Analysis. 2015. Disponível em: $<$ http:// cran.r-project.org/web/packages/nlstools/nlstools. pdf> Acesso em: 17 mar. 2015.

BREUSCH, T.S.; PAGAN, A.R. A simple test for heterocedasticity and random coefficiente variation. Econometrica, New York, v.47, n.5, 1287-1294, 1979.

CARNEIRO, A.P.S. et al. Identidade de modelos não lineares para comparar curvas de crescimento de bovinos da raça Tabapuã. Pesquisa Agropecuária Brasileira, Brasília, v.49, n.1, p.57-62, 2014.

CARVALHO, N.M.; NAKAGAWA, J. Sementes: ciência, tecnologia e produção. 4. ed. Jabotical: FUNEP, 2000. 588 p.

DRAPER, N.R.; SMITH, H. Applied regression analysis. $3^{\text {rd }}$ ed. New York: JonhWiley, 1998.

DURBIN, J.; WATSON, G.S. Teste de correlação serial em mínimos quadrados de regressão I. Biometrika, London, v.37, p.409-428, 1950.

FERNANDES, T.J.; PEREIRA, A.A.; MUNIZ, J.A. Seleção de modelos não-lineares para a descrição de curvas de crescimento do fruto do cafeeiro. Coffee Science, Lavras, v.9, n.2, p.207-215, 2014.

FILGUEIRAS, H.; MOSCA, J.; ALVES, R.; MENEZES, J. Cashew apple for fresh consumption: Research on harvest and postharvest handling technology in Brazil. Acta Horticulturae, The Hague, v.485, n.1, p.55-160,1999

FILHO SOUZA, M.M.S; MA1A, G.A.; ORIÃ, H.F.; OLIVEIRA, G.S.; FIGUEIREDO, R.W Características químicas da amêndoa da Castanha de diferentes clones do caju. Revista Ciência Agronômica, Fortaleza, v.20, p.101-104, 1989.

GAZZOLA, J.; GAZZOLA, R.; MOTTA, C.H.A amêndoa da castanha de caju: composição e importância dos ácidos graxos-produção e comércio mundiais. In: CONGRESSO DA SOBER, QUESTÕES AGRÁRIAS, EDUCAÇÃO NO CAMPO E DESENVOLVIMENTO, 44., 2006, Florianópolis. Anais... Florianópolis: Sociedade Brasileira de Economia e Sociologia Rural, 2006. 
GUEDES, M.H.P. et al. Estudo da curva de crescimento de cordeiros das raçãs Santa Inês e Bergamácia considerando heterogeneidade de variâncias. Revista Ciência e Agrotecnologia, Lavras, v.28, n.2, p.381-388, 2004.

HOTHORN, T.; ZEILEIS, A.; FAREBROTHER, R.W.; CUMMINS, C., MILLO, G.; MITCHELL, D. Package 'Imtest'. Testing linear regression models. 2014. Disponível em: $<\underline{\text { http://cran.r-roject. }}$ org/web/packages/lmtest/lmtest.pdf $>$ Acesso em: 17 mar. 2015

MAZZINI, A.R.A. et al. Análise da curva de crescimento de Novilho Hereford. Ciencia e Agrotecnologia, Lavras, v.27, n.5, p.105-112, 2003.

MAZZINI, A.R.A. et al. Curva de crescimento de novilhos Hereford: heterocedasticidade e resíduos autoregressivos. Ciência Rural, Santa Maria, v.35, n.2, p.422-427, 2005.

MENDES, P.N. et al. Modelo logístico difásico no estudo do crescimento de fêmeas de raça Hereford. Ciência Rural, Santa Maria, v.38, n.7, p.1984-1990, 2008.

MENDONÇA, W.; MEDEIROS, L.F. Cultura do cajueiro, do coqueiro e do mamoeiro. Mossoró: Universidade Federal Rural do Semiárido, 2011.

MORETTIN, P.A.; TOLOI, C.M de C. Previsão de séries temporais. 3.ed. São Paulo: Atual, 2004. $436 \mathrm{p}$.

PAIVA, F.F.A. et al. Aproveitamento industrial do caju. Fortaleza: EMBRAPA-CNPAT, 2000. 73p.

PARENTO, J.I.G.; SANTOS, J.H.R. A importância da cultura do cajueiro em especial para o Nordeste brasileiro. Correio Agrícola, São Paulo, p.42-45, 1970.

PEREIRA, J.M.; MUNIZ, J.A.; SILVA, C.A. Nonlinear models top redict Nitrogen mineralization in na oxisol. Scientia e Agricola, Piracicaba, v.62, n.4, p.395-400, 2005.
PRADO, T.K.L. et al. Ajuste do modelo logístico na descrição do crescimento de frutos de coqueiro anão por meio de algoritmos iterativos MCMC. Revista Brasileira de Biometria, São Paulo, v.31, n.2, p.216-232, 2013.

PRADO, T.K.L.; SAVIAN, T.V.; MUNIZ, J.A. Ajuste dos modelos Gompertz e Logístico aos dados de crescimento de frutos de coco anão verde. Ciência Rural, Santa Maria, v.43, n.5, p.803-809, 2013.

PRATT, H.; MENDONZA, D. Influence of nut removal on growth ripening of the Cashew apple. Journal of the American Society of Horticultural Science, Alexandria, v.105, n.4, p.540-542, 1980.

R DEVELOPMENT CORE TEAM. R: a language and environment for statistical computing. Vienna: R Foundation for Statistical Computing, 2012. Disponível em: $<$ http://www.r-project.org $>$. Acesso em: 17 maio 2013.

SANTOS, V.R. et al. Crescimento e produtividade agrícola de cana-de-açucar em diferentes frutos de fósforo. Revista Brasileira de Engenharia Agrícola e Ambiental, Campina Grande, v.13, n.4, p.389-396, 2009.

SAVIAN, T.V.; MUNIZ, J.A. A Study of in situ degradability: Heterogeneity of variances and correlated errors. Scientia Agricola, Piracicaba, v.64, p.548-554, 2007.

SEBRAE - Serviço Brasileiro de Apoio a Micro e Pequenas Empresas. Estudo sectorial cajucultura. Ceára, 2005. 44 p.

SHAPIRO, S.S.; WILK, M.B. An analysis of variance test for normality. Biometrika, Cambridge, v.52, n.3, p.591-611, 1965.

SOUZA, G. da S. Introdução aos modelos de regressão linear e não linear. Brasília: Embrapa, 2007. 505 p.

SOUZA. E.M. de et al. Modelagem não linear da extração de zinco de um solo tratado com Iodo de Esgoto. Acta Scientiarum: Techology, Maringá, v.32, n.2, p.193-199, 2010. 
SPIESS, A. Package 'qpcR'. Modelling and analysis of real-time PCR data. 2014. Disponível em: $<\underline{\text { http:// }}$ cran.r-project.org/web/packages/qpcR/qpcR.pdf $>$. Acesso em: 17 mar. 2015

TERRA, M.F.; MUNIZ, J.A.; SAVIAN, T.V. Ajuste dos modelos Logístico e Gompertz aos dados de crescimento de frutos de Tamareira anã. Magistra, Cruz das Almas, v.22, n.1, p.1-7, 2010.
TSOULARIS, A.; WALLACE, J. Analysis of logistic growth models. Mathematical Biosciences, Chicago, v.179, n.1, p.21-55, 2002.

YAHIA, E.M. Postharvest biology and tecnology of tropical and subtropical fruits. Cambridge: Woodhead, 2001. v.2, 500 p. 